\title{
Effects of Interleukin-3 on Hematopoietic Recovery after 5-Fluorouracil or Cyclophosphamide Treatment of Cynomolgus Primates
}

\author{
Alfred P. Gillio, Christina Gasparetto, Joseph Laver, Miguel Abboud, Mary Ann Bonilla, \\ Marc B. Garnick, " and Richard J. O'Reilly \\ Department of Pediatrics and Bone Marrow Transplantation, Memorial Sloan-Kettering Cancer Center,
} New York 10021; and *Genetics Institute, Cambridge, Massachusetts 02140

\begin{abstract}
Interleukin-3 (IL-3) is a hematopoietic growth factor that supports the growth of early hematopoietic progenitors in vitro. In vivo administration of recombinant human interleukin-3 (rhIL-3) to normal primates results in a modest and delayed leukocytosis secondary to increases in neutrophils, basophils, and eosinophils. We postulated that the effects of rhIL-3 might be more pronounced in hematologically stressed primates, and therefore administered rhIL-3 to primates after intensive myelosuppressive therapy. Primates received either cyclophosphamide (CPM) at $60 \mathrm{mg} / \mathrm{kg}$ or 5 -fluorouracil (5-FU) at $75 \mathrm{mg} / \mathrm{kg}$ i.v. on two consecutive days. Subsequently, rhIL-3 was administered intravenously or subcutaneously at 20 $\mu \mathrm{g} / \mathrm{kg}$ per $\mathrm{d}$ for $14 \mathrm{~d}$. Compared to controls, all rhIL-3 treated primates experienced higher absolute neutrophil count (ANC) nadirs and dramatic decreases in the period of severe neutropenia (ANC < 500) after myelosuppressive therapy. RhIL-3 administration resulted in a significant basophilia and eosinophilia, which resolved after discontinuation of the drug. RhIL-3 did not enhance erythroid recovery. Platelet recovery was earlier in rhIL-3-treated animals. However, variations in the platelet recovery observed in control animals, precluded accurate estimation of this effect or its significance. Our results indicate that the administration of rhIL-3 following intensive myelosuppressive therapy dramatically enhances myeloid recovery and ablates the predicted period of prolonged severe neutropenia. (J. Clin. Invest. 1990. 85:1560-1565.) interleukin-3 $\bullet$ cytokine $\bullet$ neutropenia $\bullet$ myelosuppression • eosinophilia $\bullet$ basophilia
\end{abstract}

\section{Introduction}

Interleukin-3 is a hematopoietic growth factor produced by activated $T$ lymphocytes (1). The recent cloning of the human IL-3 gene (2), and subsequent production of purified recombinant human interleukin-3 (rhIL-3) ${ }^{1}$ has enabled investigators

Address reprint requests to Dr. Gillio, Bone Marrow Transplant Service, 1275 York Avenue, Box 538, New York, NY 10021.

Received for publication 9 October 1989 and in revised form 18 December 1989.

1. Abbreviations used in this paper: $\mathrm{ABC}$, absolute basophil count; AEC, absolute eosinophil count; ANC, absolute neutrophil count; CPM, cyclophosphamide; 5-FU, 5-fluorouracil; GM-CSF, granulocyte macrophage-colony stimulating factor; rhIL-3, recombinant human IL-3.

J. Clin. Invest.

(C) The American Society for Clinical Investigation, Inc.

0021-9738/90/05/1560/06 \$2.00

Volume 85, May 1990, 1560-1565 to define the effects of rhIL-3 on human bone marrow progenitor cells in vitro (3-8). In serum-free bone marrow culture, rhIL-3 supports the growth of early hematopoietic progenitors and synergistically acts with more restricted cytokines, such as erythropoietin or granulocyte colony-stimulating factor (GCSF), in promoting the formation of erythroid, myeloid, mixed, and megakaryocytic colonies (3-5). In bone marrow suspension cultures, IL-3 is a potent stimulator of eosinophil and basophil differentiation (6-8).

The effects of rhIL-3 administration on hematopoiesis in normal healthy primates has also been recently described $(9$, 10). rhIL-3 treatment resulted in a modest and delayed leukocytosis secondary to increases in neutrophils, basophils, and eosinophils. In addition, increases in corrected reticulocyte counts and variable increases in platelet counts were observed.

Although the hematopoietic effects of rhIL-3 in the intact primate are not as impressive as those of the more lineage-restricted cytokines such as G-CSF (11) or granulocyte-macrophage colony-stimulating factor (GM-CSF) (12), we postulated that the effects of rhIL-3 might be more pronounced in hematologically stressed animals. In this study, rhIL-3 was administered to cynomolgus primates after myelosuppressive chemotherapy. We report that rhIL-3 administered to cynomolgus primates post-myelosuppressive therapy ablates the predicted period of prolonged severe neutropenia.

\section{Methods}

Animals. Healthy young juvenile male cynomolgus primates (Macaca fascicularis) (Hazelton Research Animals, Reston, VA) were maintained according to National Institutes of Health (NIH) guidelines for the use and care of laboratory animals. Study protocols were approved by the Memorial Sloan-Kettering Cancer Center Institutional Animal Use and Care Committee. Before all manipulations, primates were anesthetized with ketamine hydrochloride (Parke-Davis Co., Morris Plains, NJ). Indwelling intravenous Broviac catheters (Quinton Instruments, Seattle, WA) were placed sterilely in the jugular veins of the animals before myelosuppressive treatment. The indwelling catheters connected to intravenous tubing were threaded through durable nylon vests worn by the animals and then through a flexible steel coil that served as a tether allowing the animals to roam freely in their cages. After myelosuppressive treatment (as described below), animals were treated aggressively with parenteral fluids, antibiotics, and irradiated blood products. Animals received whole blood transfusions from previously crossmatched donors for hemoglobin values $<9 \mathrm{~g} / \mathrm{dl}$.

Interleukin-3. Recombinant human interleukin-3 (Genetics Institute, Cambridge, MA) expressed in Escherichia coli was purified as previously described (9). The purified rhIL-3 had a specific activity of $4.3 \times 10^{6} \mathrm{U} / \mathrm{mg}$ measured in the chronic myelogenous leukemia blast proliferation assay (2) and $<1 \mathrm{U}$ of endotoxin per milligram as measured by the Limulus amebocyte lysate assay (Whittaker Bioproducts, Walkersville, MD). Before administration, rhIL-3 was diluted to the appropriate concentration in saline containing $0.1 \%$ heat inactivated autologous serum. 
Treatment protocols. Animals received either cyclophosphamide $(\mathrm{CPM})$ at $60 \mathrm{mg} / \mathrm{kg}$ or 5 -fluorouracil (5-FU) at $75 \mathrm{mg} / \mathrm{kg}$ via intravenous bolus on two consecutive days. CPM-treated animals received two cycles of chemotherapy separated by a 32-d interval. 5-FU-treated animals were able to tolerate only one cycle of chemotherapy due to gastrointestinal toxicity. $24 \mathrm{~h}$ after the last dose of each cycle of chemotherapy, rhIL-3 treatment was initiated at $20 \mu \mathrm{g} / \mathrm{kg}$ per d for 14 consecutive days. CPM-treated animals received rhIL-3 by continuous intravenous infusion utilizing an external calibrated balloon pump (Travenol Laboratories, Deerfield, IL). However, because of the difficulty in maintaining two patent central lines in each animal, in the 5-FU trial, the same total daily dose of rhIL-3 was divided into two equal doses and administered every $12 \mathrm{~h}$ subcutaneously. Comparison of the Donahue study (9) using the continuous intravenous route and the Meyer study (10) using the subcutaneous route show no significant differences in the hematopoietic effects observed with these two methods of administration when rhIL-3 doses were within the same range. Control animals received sham doses of normal saline.

Analysis of hematopoietic recovery and rhIL-3 toxicity. Animals were weighed, examined, and vital signs measured twice daily. Automated peripheral blood counts (Coulter S; Coulter Instruments, Hialeah, FL), manual 100 cell differentials and corrected reticulocyte counts were obtained daily for $30 \mathrm{~d}$ after each cycle of chemotherapy. In addition, serum electrolytes, biochemical profiles, and coagulation parameters were measured twice weekly during the same period.

Granulocyte-macrophage colony forming units (CFU-GM) assay. Bone marrow was aspirated from primate femurs into heparinized syringes and erythrocytes were removed by differential sedimentation in $3 \%$ gelatin. Low density bone marrow mononuclear cells were further isolated by separation on Ficoll-Hypaque, and were plated at a concentration of $1 \times 10^{5} / \mathrm{ml}$ in $35-\mathrm{mm}$ tissue culture dishes containing a 1-ml mixture of IMDM (Gibco Laboratories, Grand Island, NY), 0.36\% Agarose (FMS, Rockland, ME), 20\% fetal calf serum (HyClone Labs, Logan, UT), in the presence of rhGM-CSF $(1,000 \mathrm{U} / \mathrm{ml})(\mathrm{Ge}-$ netics Institute, Cambridge, MA). Cultures were incubated at $37^{\circ} \mathrm{C}$ in humidified $5 \% \mathrm{CO}_{2}$ in air and CFU-GM colonies/clusters were scored on day 7.

\section{Results}

$C P M$ trial. Three primates received two sequential cycles of CPM. One of the primates received rhIL-3 after each CPM cycle and two primates served as controls. The total white blood counts (WBC) of the rhIL-3-treated animal remained higher than those of the control animals throughout the study (Fig. $1 \mathrm{~A}$ ). After CPM treatment, all animals experienced a decline in WBC, which reached a nadir 6-12 d later. The control animals experienced WBC nadirs of $1.3 \times 10^{3} / \mathrm{mm}^{3}$ and $1.0 \times 10^{3} / \mathrm{mm}^{3}$ after CPM cycle 1 , and $0.9 \times 10^{3} / \mathrm{mm}^{3}$ and $0.5 \times 10^{3} / \mathrm{mm}^{3}$ following CPM cycle 2 (Table I). In contrast, the WBC nadirs of the rhIL-3 treated animal were $3.7 \times 10^{3} /$ $\mathrm{mm}^{3}$ and $3.6 \times 10^{3} / \mathrm{mm}^{3}$ following CPM cycles 1 and 2 , respectively. The absolute neutrophil counts (ANC) of the rhIL-3 treated animal also remained greater than those of the control animals throughout the study (Fig. 1 B). After each CPM cycle, the control animals developed severe neutropenia, defined as an absolute neutropenia count below $0.5 \times 10^{3}$ / $\mathrm{mm}^{3}$. The ANC nadirs of both animals were $0.4 \times 10^{3} / \mathrm{mm}^{3}$ and $0.1 \times 10^{3} / \mathrm{mm}^{3}$ after CPM cycles 1 and 2 , respectively (Table I). The duration of neutropenia in the control animals after CPM cycle 1 was 1 and $4 \mathrm{~d}$, and following CPM cycle 2, 7 and $12 \mathrm{~d}$ (Table I). The rhIL-3-treated animal did not develop severe neutropenia. The ANC remained $>1.0 \times 10^{3} / \mathrm{mm}^{3}$ after both cycles of CPM.

Qualitatively, the WBC recovery post-CPM was different between the two groups. WBC recovery in control animals was composed primarily of neutrophils, lymphocytes and monocytes, in proportions comparable to those in normal cynomolgus peripheral blood (Fig. $2 A$ ). No significant eosinophilia or basophilia was observed. In contrast, the rhIL-3-treated animal developed a marked eosinophilia and basophilia (Fig. 2 $B$ ). After each CPM cycle (Fig. 3), absolute eosinophil counts (AEC) and absolute basophil counts $(\mathrm{ABC})$ rose above the normal baseline values $\left(0-505 / \mathrm{mm}^{3}\right.$ and $0-213 / \mathrm{mm}^{3}$, respectively, based on peripheral white blood counts and differential obtained in 25 normal cynomolgus primates in our colony) by days 6-8 and reached a maximum on days $15-21$. The AEC and $A B C$ returned to normal baseline values by day 28 of each CPM cycle.

5-FU trial. 5-FU was administered to six primates. Subsequently, two of these primates (A and B) received a 14-d course of rhIL-3, and four control animals (C-F) received sham doses of normal saline over the same time period. rhIL-3-treated animals experienced a significant acceleration of both total white blood cell and neutrophil recovery after 5-FU compared with controls (Fig. 4). Control animals developed a prolonged neutropenia, lasting 18-25 d, with ANC nadirs of $0.0-0.1 \times 10^{3} / \mathrm{mm}^{3}$ (Fig. $4 \mathrm{~B}$, Table II). In contrast, both rhIL-3-treated animals experienced only one day of severe neutropenia and ANC nadirs of $0.4 \times 10^{3} / \mathrm{mm}^{3}$ (Fig. $4 \mathrm{~B}$, Table II). WBC recovery in rhIL-3-treated primates was associated with significant eosinophilia and basophilia, which was not observed in control animals. In primates $A$ and $B$, an absolute eosinophilia developed between days $11-24$, peaking on days 14 and 15 at $3.2 \times 10^{3} / \mathrm{mm}^{3}$ and $2.3 \times 10^{3} / \mathrm{mm}^{3}$, respectively (Fig. $5 \mathrm{~A}$ ). An absolute basophilia was observed in primates $A$ and $B$ on days $8-21$, reaching maximal levels on

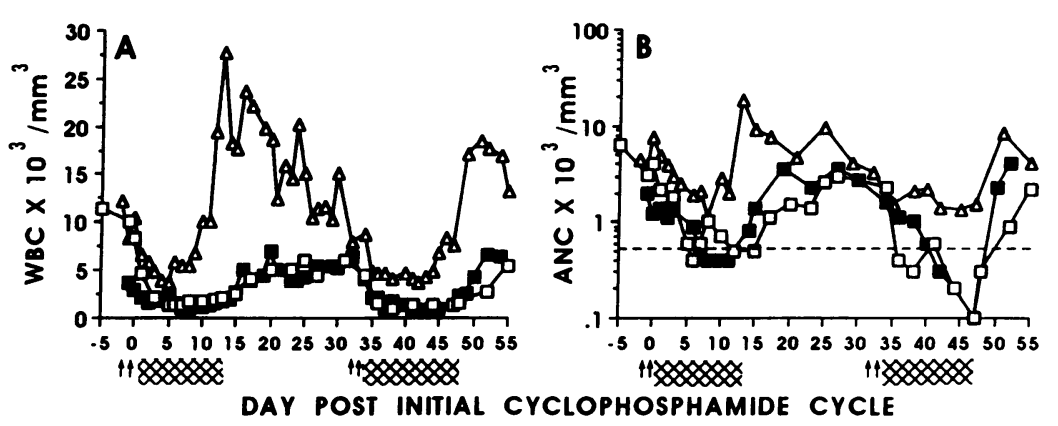

Figure 1. Time course of peripheral blood white blood count (WBC) $(A)$ and absolute neutrophil count (ANC) $(B)$ in cynomolgous primates treated with two cycles of cyclophosphamide (CPM) separated by an interval of $32 \mathrm{~d}$. A cycle consisted of CPM given at $60 \mathrm{mg} / \mathrm{kg}$ intravenously on two consecutive days (days $-1,0$ and days 32,33 -depicted by vertical arrows). After each CPM cycle, primate $(\Delta)$ received rhIL-3 via continuous intravenous infusion at $20 \mu \mathrm{g} / \mathrm{kg}$ per $\mathrm{d}$ for 14 consecutive days (days 1-14 and 34-47, depicted by crosshatched bar). Primates $(\square, \square)$ served as controls and received only sham doses of normal saline. $(B)$ Note log scale and dotted line, which depicts ANC of 500 . 
Table I. Effect of rhIL-3 Administration on Myeloid Recovery after Two Cycles of Cyclophosphamide Therapy

\begin{tabular}{|c|c|c|c|c|c|c|}
\hline \multirow[b]{2}{*}{ Primate } & \multicolumn{2}{|c|}{ WBC Nadir* } & \multicolumn{2}{|c|}{ ANC Nadir* } & \multicolumn{2}{|c|}{ Days ANC $<500$} \\
\hline & Cycle 1 & Cycle 2 & Cycle 1 & Cycle 2 & Cycle 1 & Cycle 2 \\
\hline IL-3 & 3.7 & 3.6 & 1.1 & 1.3 & 0 & 0 \\
\hline Control 1 & 1.3 & 0.9 & 0.4 & 0.1 & 1 & 7 \\
\hline Control 2 & 1.0 & 0.5 & 0.4 & 0.1 & 4 & 12 \\
\hline
\end{tabular}

* Cells $\times 10^{3} / \mathrm{mm}^{3}$.

days 12 and 15 of $4.7 \times 10^{3} / \mathrm{mm}^{3}$ and $3.6 \times 10^{3} / \mathrm{mm}^{3}$, respectively (Fig. 5 B).

The incidence of rhGM-CSF stimulated CFU-GM per 1 $\times 10^{5}$ bone marrow mononuclear cells following 5-FU with or without rhIL-3 treatment is depicted in two representative primates (Fig. 6). Animals receiving rhIL-3 experienced a more rapid recovery of CFU-GM, achieving baseline values by day 14 compared to day 21 in controls. Similar results were obtained with in vitro stimulation of CFU-GM with rhG-CSF and rhIL-3.

We observed no obvious differences in erythroid recovery post-5-FU in rhIL-3-treated versus control animals, as measured by recovery of corrected reticulocyte counts and the red cell transfusion requirements of the animals.

5-FU administration, unlike CPM, resulted in a significant depression of platelet counts below baseline levels (Fig. 7). Among the four control animals platelet counts fell from 273 to $553 \times 10^{3} / \mathrm{mm}^{3}$ on day -2 to nadir counts of $96-150$ $\times 10^{3} / \mathrm{mm}^{3}$ between days 14 and 19 (Table II). Platelet counts remained depressed greater than 30 days in two control animals (C, D). However, two controls (E, F) experienced abrupt increases in platelet counts beginning on days 17 and 15 , reaching maximum levels of 668 and $808 \times 10^{3} / \mathrm{mm}^{3}$, respectively. Nadir platelet counts in the IL-3-treated primates (A, B), of 117 and $368 \times 10^{3} / \mathrm{mm}^{3}$ occurred on days 11 and 12 , respectively (Table II). Abrupt platelet count recovery occurred in both animals beginning on days 11 and 14, resulting in maximum platelet counts of 830 and $838 \times 10^{3} / \mathrm{mm}^{3}$.

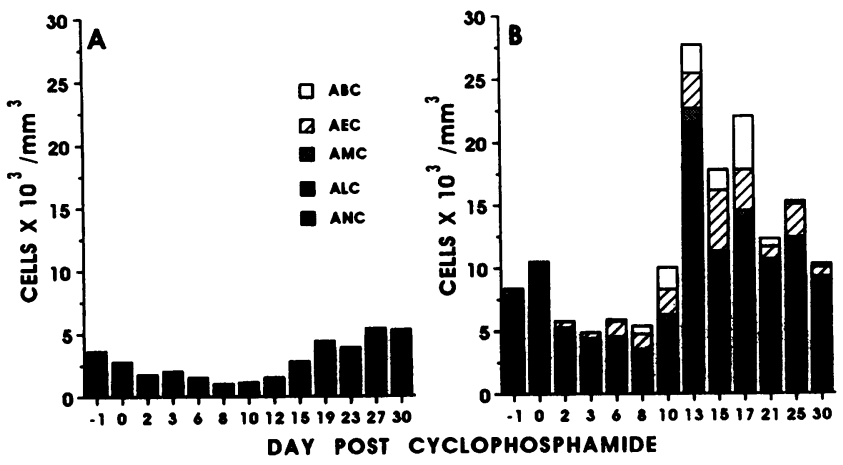

Figure 2. Time course of peripheral white blood cell differentials in primates receiving one cycle of CPM (days $-1,0) .(A)$ Differential counts from a control animal and $(B)$ differential counts from an animal who received a 14-d infusion of rhIL-3 (days 1-14).

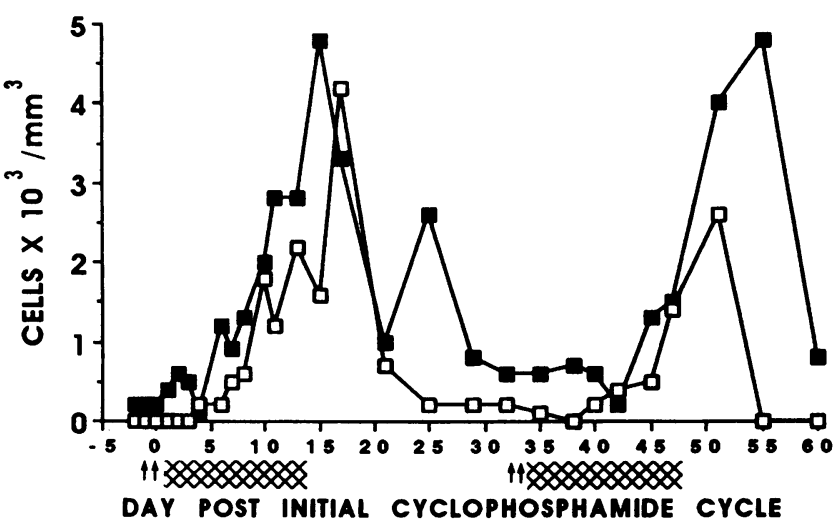

Figure 3. Time course of absolute basophil counts ( $\square$ ) and absolute eosinophil counts $(\square)$ in a primate treated with two cycles of CPM followed by $14-d$ intervals of rhIL-3.

RhIL-3 toxicity. All rhIL-3-treated primates developed localized facial and extremity swelling 8 to 11 days after the initiation of rhIL-3 treatment. The swelling was non-pitting and not associated with weight gain. Concurrently, a pruritic rash developed consisting of $1-2 \mathrm{~cm}$ yellow to pink indurated papules. Biopsy of the affected dermis revealed perivascular infiltration of neutrophils and eosinophils. No life-threatening allergic symptomatology was noted. The rash and swelling resolved within one week of rhIL-3 discontinuation. All rhIL-3 treated primates also developed mild splenomegaly without hepatomegaly. No hepatic or renal toxicity was observed.

\section{Discussion}

To examine the effect of rhIL-3 on hematopoiesis in hematologically stressed primates, we administered two different and diverse cytotoxic agents: cyclophosphamide, an alkylating agent that is cycle nonspecific and relatively platelet sparing; and 5-fluorouracil, an antimetabolite, which is cycle specific and at doses employed in this study extremely myelosuppressive. In mice, this dose of 5-FU has been shown to spare only those primitive marrow progenitors with high proliferative potential $(13,14)$. We attempted to administer two cycles of

Table II. Effect of rhIL-3 Administration on Myeloid and Platelet Recovery after 5-Fluorouracil Therapy

\begin{tabular}{lccc}
\hline \multicolumn{1}{c}{ Primate } & ANC Nadir* & Days ANC $<500$ & Nadir platelet count (Day) \\
\hline IL-3 (A) & 0.4 & 1 & $117(11)$ \\
IL-3 (B) & 0.4 & 1 & $368(12)$ \\
Control (C) & 0.0 & 20 & $96(19)$ \\
Control (D) & 0.0 & 18 & $99(14)$ \\
Control (E) & 0.1 & 25 & $105(16)$ \\
Control (F) & 0.0 & 19 & $150(14)$
\end{tabular}

* Cells $\times 10^{3} / \mathrm{mm}^{3}$.

$\ddagger$ Day post 5-FU of nadir platelet count. 


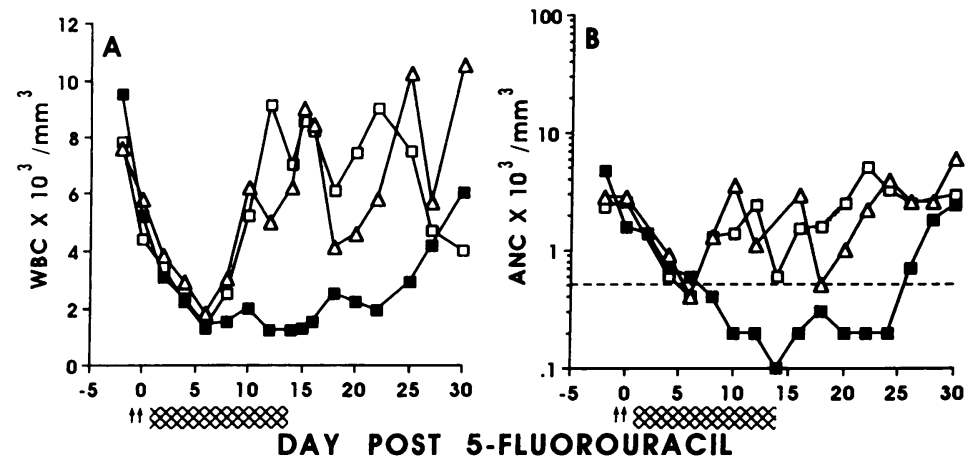

Figure 4. Time course of white blood counts $(A)$ and absolute neutrophil counts $(B)$ in cynomolgus primates treated with 5 -FU at $75 \mathrm{mg} / \mathrm{kg}$ i.v. on two consecutive days (days $-1,0)$. After 5-FU, primates $(\square, \Delta)$ received rhIL-3 at 20 $\mu \mathrm{g} / \mathrm{kg}$ per $\mathrm{d}$ for 14 consecutive days (days $1-14$ ) subcutaneously. The white blood counts of the four control primates are averaged and depicted as $\mathbf{m}$. each chemotherapeutic agent in order to mimic pulse repetitive cytotoxic therapy as it is commonly used in human trials, however the animals receiving 5-FU were only able to tolerate one cycle of therapy due to severe gastrointestinal toxicity.

The administration of rhIL-3 following both cytotoxic agents clearly resulted in a marked enhancement of myeloid recovery. In both experiments, rhIL-3-treated primates compared to controls experienced higher absolute neutrophil count nadirs and an ablation or a significant decrease in the period of severe neutropenia following myelosuppression. In addition to changes in the peripheral counts, we also observed evidence of earlier recovery of myeloid progenitors in sequential clonal assays of bone marrow in rhIL-3-treated primates compared to controls following 5-FU treatment.

In the CPM experiment, rhIL-3 administration after two consecutive cycles of high-dose alkylator therapy did not lead to demonstrable enhanced depletion of marrow progenitors capable of reconstituting hematopoiesis or significantly enhance the sensitivity of early hematopoietic progenitors to subsequent cytotoxic therapy, since the hematopoietic response seen after the second cycle of CPM was equivalent or even perhaps better, than that observed after the first CPM cycle.

In in vitro studies, rhIL-3 when combined with erythropoietin exhibits substantial burst-promoting activity (15). However, we could not demonstrate a stimulatory effect of rhIL-3 on the erythroid lineage in the 5-FU experiments. It is possible that the necessary transfusion of whole blood to the primates blunted an erythroid response. Furthermore, our measures of red blood cell production, which included only corrected reticulocyte counts and transfusion requirements, may not have been sufficiently sensitive to discern alterations in erythroid precursor recovery in animals undergoing daily blood samplings and experiencing gastrointestinal blood losses, thereby requiring varying amounts of transfusion support.

In vitro serum-free marrow culture studies have also demonstrated that rhIL-3 is a megakaryocyte colony stimulating factor $(16,17)$. In this study, the effect of rhIL-3 on megakaryopoiesis could only be assessed in the 5-FU experiments. In the 5-FU trial, the four control animals exhibited two very different patterns of platelet recovery. Primates $C$ and $D$ displayed very prolonged delays in platelet recovery, while primates $\mathrm{E}$ and $\mathrm{F}$ experienced abrupt elevations in platelet counts to twice baseline levels on days 15 and 17 . This latter pattern of recovery was exhibited by the rhIL-3 treated primates. However, it occurred earlier, on days 11 and 14, suggesting that IL-3 may also stimulate more prompt recovery of megakaryopoiesis in vivo. Additional experiments are in progress to accurately assess differences in platelet recovery times and their significance.

In bone marrow suspension culture systems, rhIL-3 induces the growth of basophils and the production of intracellular histamine (18). The administration of rhIL-3 to normal rhesus primates results in dramatic increases in blood eosinophils and basophils and a dose-dependent increase in intracellular histamine and to a less extent plasma histamine (10). Whether rhIL-3 induces blood basophils to release intracellular histamine is currently debated in the literature. Valent et al.

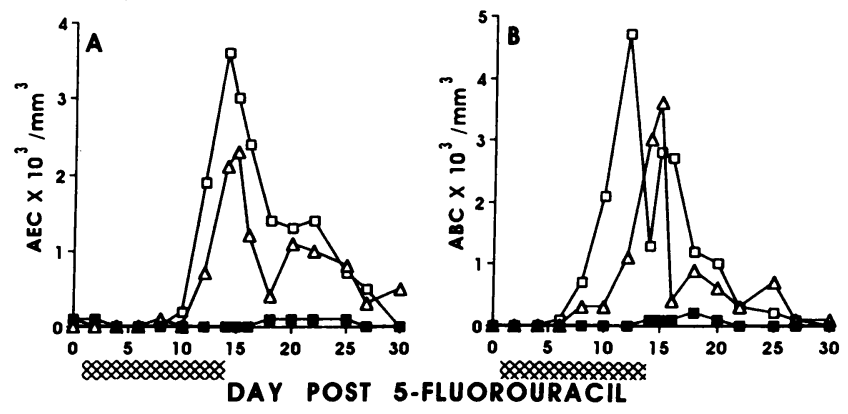

Figure 5. Time course of absolute eosinophil counts $(A)$ and absolute basophil counts $(B)$ in primates treated with 5 -FU. Primates $(\square, \Delta)$ received rhIL-3 as previously described. Average counts of the four control primate are depicted as $\mathbf{a}$.

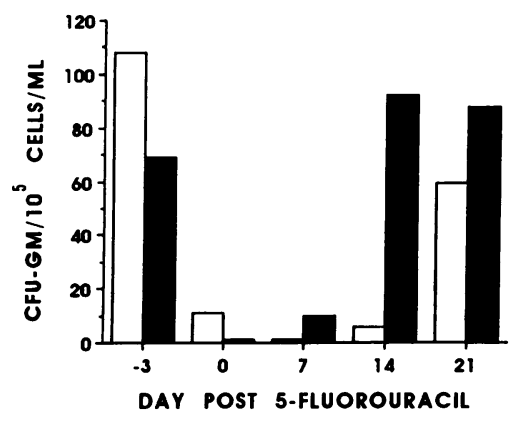

Figure 6. Incidence of rhGM-CSF stimulated CFU-GM per $1 \times 10^{5}$ bone marrow mononuclear cells obtained from two representative primates, before 5-FU (day -3), and then weekly post 5-FU. The closed bars depict data from a primate who received rhIL-3 post 5-FU and open bars data from a control animal. 


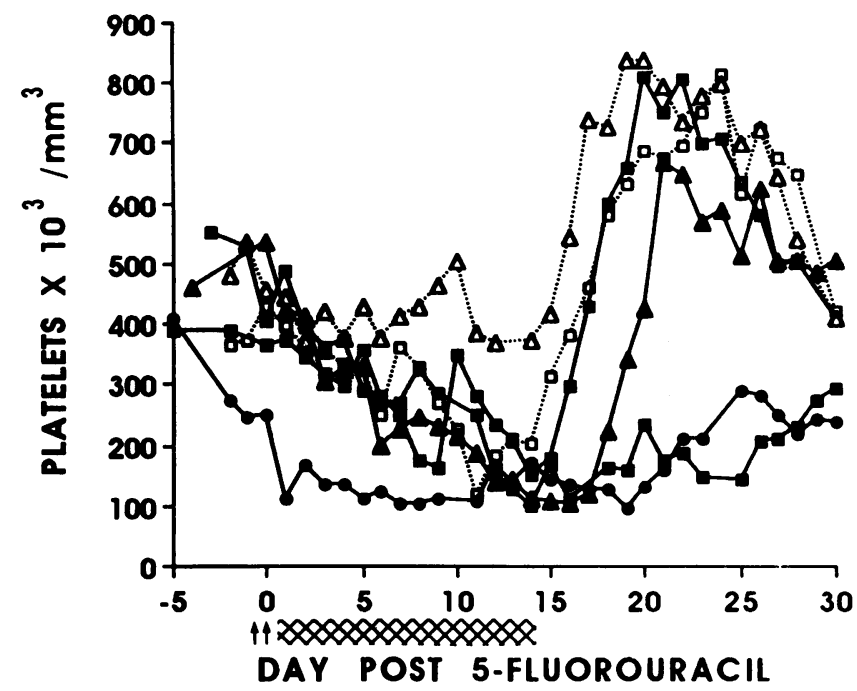

Figure 7. Time course of platelet count recovery in primates treated with 5-FU as previously described. Open symbols $(\Delta, \square)$ depict animals treated post-5-FU with rhIL-3, and closed symbols depict control animals.

(18) have reported that rhIL-3 is not a histamine releasing factor, while Haak-Frendscho et al. (19) have reported the converse. In this study, we observed significant basophilia and eosinophilia in all rhIL-3-treated primates. This basophil increase if also associated with an increase in plasma histamine, would explain the side effects observed in the rhIL-3-treated animals. All animals developed localized swelling and a pruritic rash concomitant with the increase in basophil numbers, which completely resolved as basophil numbers returned to normal levels. Biopsy of the rash was consistent with an urticarial lesion. It is important to note, however, that in this study and in the previously reported in vivo rhIL-3 primate trials $(9$, 10), animals with abnormally high basophil counts have not developed any serious or life-threatening allergic symptoms.

These results strongly encourage the testing of rhIL-3 in human clinical trials. rhIL-3, like G-CSF and GM-CSF, clearly has stimulating effects on myeloid recovery postcytotoxic chemotherapy. It is also possible that IL-3 will be more effective than G-CSF and GM-CSF in stimulating hematopoietic recovery in patients treated with myeloablative dose of agents such as 5-FU, which effect early marrow progenitors. Further studies are required to accurately assess the effects of rhIL-3 on red cell and platelet recovery. However, our results suggest that IL-3 may also stimulate earlier recovery of megakaryopoiesis. To better define these issues, we are currently evaluating the effects of rhIL-3 on hematopoietic recovery following ablative doses of total body irradiation and autologous transplants of deescalating doses of marrow progenitors.

\section{Acknowledgments}

We thank G. Eneman, K. Fliszar, G. Gonzalez, K. Pabon, and R. Primm for excellent animal care and D. Cheung for assistance with the blood counts. We also thank C. Thomas and A. Campbell for assistance in preparation of this manuscript.
This work was supported in part by Genetics Institute and research grants from the National Institutes of Health (CA09512-04, CA23766), the Clinical Scholars National Research Service Award, Vincent Astor Fund, Toys " $Я$ " Us Foundation, Laura Rosenberg Memorial Foundation, and the Andrew Gaffney Foundation. The rhIL-3 used in these studies was provided by Genetics Institute.

\section{References}

1. Niemeyer, C. M., C. A. Sieff, B. Mathey-Prevot, J. Z. Wimperis, B. E. Bierer, S. C. Clark, and D. G. Nathan. 1989. Expression of human interleukin-3 (multi-CSF) is restricted to human lymphocytes and T-cell tumor lines. Blood. 73:945-951.

2. Yang, Y.-C., A. B. Ciarletta, P. A. Temple, M. P. Chung, S. Kovacic, J. S. Witek-Giannotti, A. C. Leary, R. Kriz, R. E. Donahue, G. G. Wong, and S. C. Clark. 1986. Human IL-3 (multi-CSF): identification by expression cloning of a novel hematopoietic growth factor related to murine IL-3. Cell. 47:3-10.

3. Sonoda, Y., Y.-C. Yang, G. G. Wong, S. C. Clark, and M. Ogawa. 1988. Analysis in serum-free culture of the targets of recombinant human hemopoietic growth factors. Interleukin-3 and granulocyte/macrophage-colony-stimulation factor are specific for early developmental stages. Proc. Natl. Acad. Sci. USA. 85:4360-4364.

4. Sieff, C. A., S. C. Ekern, D. G. Nathan, and J. W. Anderson. 1989. Combinations of recombinant colony-stimulating factors are required for optimal hematopoietic differentiation in serum-deprived culture. Blood. 73:688-693.

5. Bot, F. J., L. van Eijk, P. Schipper, and R. Lowenberg. 1989. Effects of human interleukin-3 on granulocytic colony-forming cells in human bone marrow. Blood. 73:1157-1160.

6. Saito, H., K. Hatake, A. M. Dvorak, K. M. Leiferman, A. D. Donnenberg, N. Arai, K. Ishizaka, and T. Ishizaka. 1988. Selective differentiation and proliferation of hematopoietic cells induced by recombinant human interleukins. Proc. Natl. Acad. Sci. USA. 85:22882292.

7. Ottmann, O. G., M. Abboud, K. Welte, L. M. Souza, and L. M. Pelus. 1989. Stimulation of human hematopoietic progenitor cell proliferation and differentiation by recombinant human interleukin-3. Comparison and interactions with recombinant human granulocytemacrophage and granulocyte colony-stimulating factors. Exp. Hematol. 17:191-197.

8. Abboud, M., and M. A. S. Moore. 1989. Recombinant human IL-3 is a basophil/mast cell growth factor. Exp. Hematol. 16:489. (Abstr.)

9. Donahue, R. E., J. Seehre, M. Metzger, D. Lefebvre, B. Rock, S. Carbone, D. G. Nathan, M. Garnick, P. K. Sehgal, D. Laston, E. LaVallie, J. McCoy, P. F. Schendel, C. Norton, K. Turner, Y.-C. Yang, and S. C. Clark. 1988. Human IL-3 and GM-CSF act synergistically in stimulating hematopoiesis in primates. Science (Wash. DC). 241:1820-1823.

10. Mayer, P., P. Valent, G. Schmidt, and P. Bettelheim. 1989. The in vivo effects of recombinant human interleukin-3: demonstration of basophil differentiation factor, histamine-producing activity, and priming of GM-CSF-responsive progenitors in nonhuman primates. Blood. 74:613-621.

11. Welte K., M. A. Bonilla, A. P. Gillio, T. C. Boone, G. K. Potter, J. L. Gabrilove, M. A. S. Moore, R. J. O'Reilly, and L. M. Souza. 1987. Recombinant human granulocyte colony-stimulating factor. Effects on hematopoiesis in normal and cyclophosphamide-treated primates. J. Exp. Med. 165:941-948.

12. Donahue, R. E., E. A. Wang, D. K. Stone, R. Kamen, G. G. Wong, P. K. Sehgal, D. G. Nathan, and S. E. Clark. 1986. Stimulation of hematopoiesis in primates by continuous infusion of recombinant human GM-CSF. Nature (Lond.). 321:872-875.

13. Hodgson, G. S., T. R. Bradley, and J. M. Radley. 1982. The 
organization of hematopoietic tissue as inferred from the effects of 5-fluorouracil. Exp. Hematol. 10:26-35.

14. Van Zant, G. 1984. Studies of hematopoietic stem cells spared by 5-fluorouracil. J. Exp. Med. 159:679-690.

15. Sonoda, Y., Y.-C. Yang, G. G. Wong, S. C. Clark, and M. Ogawa. 1988. Erythroid burst-promoting activity of purified recombinant human GM-CSF and interleukin-3. Studies with anti-GM-CSF and anti-IL-3 sera and studies in serum-free cultures. Blood. 72:13811386.

16. Bruno, E., M. E. Miller, and R. Hoffman. 1989. Interacting cytokines regulate in vitro human megakaryocytopoiesis. Blood. 73:671-677.
17. Briddell, R. A., J. E. Brandt, J. E. Straneva, E. F. Srour, and R. Hoffman. 1989. Characterization of the human burst-forming unitmegakaryocyte. Blood. 74:145-151.

18. Valent P., G. Schmidt, J. Besemer, P. Mayer, G. Zenke, E. Liehl, W. Hinterberger, K. Lechner, D. Maurer, and P. Bettelheim. 1989. Interleukin-3 is a differentiation factor for human basophils. Blood. 73:1763-1769.

19. Haak-Frendscho, M., N. Arai, K.-I. Arai, M. L. Baeza, A. Finn, and A. P. Kaplan. 1988. Human recombinant granulocyte-macrophage colony-stimulating factor and interleukin-3 cause basophil histamine release. J. Clin. Invest. 82:17-20. 